\title{
A mobile assisted coverage hole patching scheme based on particle swarm optimization for WSNs
}

Article

Accepted Version

Wang, J., Ju, C., Kim, H.-j., Sherratt, R. S. and Lee, S. (2019) A mobile assisted coverage hole patching scheme based on particle swarm optimization for WSNs. Cluster Computing, 22 (1). pp. 1787-1795. ISSN 1386-7857 doi:

https://doi.org/10.1007/s10586-017-1586-9 Available at https://centaur.reading.ac.uk/74429/

It is advisable to refer to the publisher's version if you intend to cite from the work. See Guidance on citing.

To link to this article DOI: http://dx.doi.org/10.1007/s10586-017-1586-9

Publisher: Springer

All outputs in CentAUR are protected by Intellectual Property Rights law, including copyright law. Copyright and IPR is retained by the creators or other copyright holders. Terms and conditions for use of this material are defined in the End User Agreement.

www.reading.ac.uk/centaur 
Central Archive at the University of Reading

Reading's research outputs online 


\title{
Full-Text version
}

Title:

\author{
A Mobile Assisted Coverage Hole Patching Scheme based on Particle Swarm Optimization \\ for WSNs
}

Authors:

Jin Wang, School of Information Engineering, Yangzhou University, Yangzhou, China, and Key Lab of Broadband Wireless Communication and Sensor Network Technology, Nanjing University of Posts and Telecommunications, Ministry of Education, Nanjing, China, jinwang@yzu.edu.cn

Chunwei Ju, School of Information Engineering, Yangzhou University, Yangzhou, China, jvchunwei@163.com

Hye-jin Kim, Business Administration Research Institute, Sungshin W. University, Korea, hye-jinkim@hotmail.com

R. Simon Sherratt, Department of Biomedical Engineering, the University of Reading, UK, sherratt@ieee.org

Sungyoung Lee, Computer Engineering Department, Kyung Hee University, Suwon, Korea, sylee@oslab.khu.ac.kr

\begin{abstract}
:
Wireless sensor networks (WSNs) have drawn much research attention in recent years due to the superior performance in multiple applications, such as military and industrial monitoring, smart home, disaster restoration etc. In such applications, massive sensor nodes are randomly deployed and they remain static after the deployment, to fully cover the target sensing area. This will usually cause coverage redundancy or coverage hole problem. In order to effectively deploy sensors to cover whole area, we present a novel node deployment algorithm based on mobile sensors. First, sensor nodes are randomly deployed in target area, and they remain static or switch to the sleep mode after deployment. Second, we partition the network into grids and calculate the coverage rate of each grid. We select grids with lower coverage rate as candidate grids. Finally, we awake mobile sensors from sleep mode to fix coverage hole, particle swarm optimization (PSO) algorithm is used to calculate moving position of mobile sensors. Simulation results show that our algorithm can effectively improve the coverage rate of WSNs.
\end{abstract}

Publication: Cluster Computing

Publisher: $\quad$ Springer

ISSN: $\quad$ ISSN: 1386-7857 (Print) 1573-7543 (Online)

Volume: not yet assigned

Issue: $\quad$ not yet assigned

Accepted Date: $\quad 7^{\text {th }}$ December 2017

pp.: not yet assigned

DOI: $\quad$ not yet assigned

Key words: Wireless Sensor Network; Particle Swarm Optimization (PSO); Coverage; Sensor Deployment 


\section{Introduction}

With the rapid development of information technologies, wireless sensor networks (WSNs) are becoming a very hot research area in recent years [1,2]. Owing to their characteristics such as low cost, robustness, local collaboration and highly adaptability, WSNs can be applied to numerous applications such as military surveillance, target monitoring and detection, smart home and environment monitoring etc.[3]. For large scale WSNs, sensor nodes are usually massively and randomly deployed in target area [4], which can result in uneven deployment of nodes and add the network cost. Thus node deployment strategies play a vital role under these circumstances.

Coverage control mainly focuses on optimizing nodes deployment algorithms and adjusting nodes positions to construct network with limited resources, it aims to guarantee the Quality of Service (QoS) in monitoring Region of Interest (RoI) [5]. However in traditional node deployment algorithms, sensor nodes are randomly deployed in the RoI and this can give rise to the coverage hole problem, which will influence network performance of WSNs. Thus efficient node deployment algorithms are required to solve this problem.

Mobile assist node deployment scheme is a feasible and effective solution to eliminate such coverage hole problem [6]. With the aim to fully cover the whole network, mobile sensor nodes are utilized in WSNs with static sensor nodes. For mobile sensor nodes, they can move to the coverage hole to fix it [7]. In recent research, swarm intelligence algorithm has become more and more popular and can be utilized to find the moving position for mobile sensor nodes [9].

In this paper, we propose a mobile assisted node deployment algorithm based on particle swarm optimization. Firstly, all sensor nodes are randomly deployed in the target area, and they remain static after deployment. Static sensors switch to the active mode in the initial phase. In the meanwhile, mobile sensors switch to sleep mode. Then, we partition the network into several grids and select grids with low coverage rate as candidate grids. Finally, we awake mobile sensors from sleep mode to patch coverage hole.

The remaining of this paper is organized as follows. Section 2 covers some related works. Section 3 shows our system model and Section 4 presents our proposed algorithm in detail. Simulation results are presented in Section 5. Section 6 gives some discussion and open research issues and Section 7 concludes this paper.

\section{Related Work}

Many researches have been conducted on coverage control issue in WSNs. The authors in [7] studied efficient node deployment algorithms in mobile WSNs to improve the network coverage rate. Based on the existing coverage holes in the network, their proposed algorithm locates the suitable position for each mobile sensor. They utilized multiplicatively weighted Voronoi (MW-Voronoi) diagram to find coverage holes and the authors therein also introduced three algorithms under different situations.

In [8], the authors discussed issues during designing movement-assisted sensor deployment algorithms. They conducted a survey about the movement-assisted sensor deployment algorithms. Besides, they also defined six classes of approaches and each approach has specific nodes relocate rules. In each class, various deployment algorithms are discussed and authors gave a comparison for these algorithms. Furthermore, authors presented some open topics in this research area.

The authors in [9] concentrated on target coverage problems, and they aimed to achieve two objectives in this paper. First, they found the optimal deployment locations for sensor nodes in order to prolong the network lifetime. Second, they schedule these sensor nodes to maximize nodes' lifetime. Meanwhile, they introduced artificial bee colony algorithm during sensor deployment and particle swarm optimization algorithm during nodes scheduling phase.

Senouci et al. presented a mobile-assisted energy hole detection and healing algorithm in [10]. Effective coverage is essential since the energy hole problem is unavoidable under nature environment. Before the authors tried to solve the problem, they discussed the drawbacks of existing coverage healing algorithms. Then they gave four key elements of effective coverage in WSNs. By combining these elements, they proposed a comprehensive algorithm for coverage detection and healing algorithm for mobile WSNs. Simulation results show the superiority of their proposed algorithm.

Liao et al. proposed a target coverage algorithm to minimize sensor movement [11]. The authors found that many researchers introduce mobile sensors to cover target area, but few of them focus on minimizing sensor movement which will consume extra energy cost for sensors. With the aim of minimizing sensors movement and improving connectivity, they solved these two sub-problems one by one. Then, they combined these two 
solutions together and these problems can be well solved.

The authors in [12] classified the node deployment methods into three categories, namely random, incremental and movement-assisted methods. Besides, they presented four different mathematical approaches and gave each approach some discussion. Furthermore they studied each algorithms' advantages and limitations.

Rakavi et al. presented a grid based mobile sensor node deployment algorithm in [13]. This algorithm combines static nodes with mobile nodes. Mobile nodes are utilized to fix coverage hole after static nodes are deployed. Target area is partition into grids, and each grid's weighted value is calculated. Target grid has small weighted value, mobile sensors move to the grid with small weight. In [14], authors used mobile sensors to fix coverage hole in WSNs. The coverage hole is shown as an irregular polygon and mobile sensors move into polygon to fix coverage hole.

Authors in [15] proposed a distributed hybrid coverage hole healing algorithms which gave a game theoretic approach. Each sensor combined physical relocation and sensing range adjustment to minimize the coverage hole. Simulation results show the superior performance of this algorithm. Dung et al. presented a coverage hole healing algorithm in literature [16]. They aimed to utilize mobile sensors to estimate coverage hole problem. Besides, they proposed a new metric to design the efficient coverage hole healing algorithm.

Zhang et al. studied barrier coverage under random deployed mobile wireless sensor networks in [17]. They concentrated on minimizing number of relocated mobile sensors and achieving k-barrier coverage with minimum energy cost. Theoretical analysis and simulation results show the superiority of their approach. In [18], the authors put research efforts on meteorological wireless sensor networks. For low cost sensors, they have advantage of quick deployment and high adaptability, but the accuracy of measured data is easily affected by external environment. Through previous research, the authors found a close relationship between air temperature (AT) and solar radiation (SR), so they introduced BP neural network model to modify data.

The authors in [19] proposed a cluster-based data collection algorithm with multiple mobile sinks. Besides, rendezvous points (RPs) and rendezvous nodes (RNs) were utilized to achieve optimal energy efficiency. Simulation results show that network lifetime is prolonged in different situations.

To improve energy efficiency and reliability in delay tolerant WSNs, authors in [20] introduced mobile data collectors in their proposed algorithms. They studied mobile sinks and mobility models for its performance in energy efficiency and reliability. Simulation results show that their proposed data collection approach and mobility model can have better performance.

Mobile agents are widely used for collaborative processing in WSNs, but their schedule schemes still require further research effort. Authors in [21] proposed a distributed scheme to plan the moving path of agents. In [22], efficient routing strategy is presented for mobile ad hoc network. This scheme allows selection backup nodes from neighbors.

Zhu et al. [23] discussed device-free through-the-wall detection based on Wi-Fi. They provided a new human-computer interactive (HCI) method. They discussed the existing challenge in through-the-wall (TTW) human detection, and presented a reliable solution. Authors in [24] gave an unknown bugs discovery based on analysis for known patches. They proposed VulHunter for unknown vulnerabilities discovery.

\section{System Model}

\subsection{Network Model}

The network can be viewed as a directed graph $\mathrm{G}(V, E)$, where $V$ represents the set of sensor nodes. $E_{i j}$ represents the set of all links between node $i$ and node $j$.

In this paper, we consider that $M$ static sensor nodes and $N$ mobile sensor nodes are randomly deployed in $K \times K$ square sensing area, as is shown in Figure 1. Static sensor nodes are denoted as $\left\{M_{1}, M_{2}, M_{3}, \ldots, M_{m}\right\}$ and mobile sensors are denoted as $\left\{N_{1}, N_{2}, N_{3}, \ldots, N_{n}\right\}$ respectively. We consider that all static sensor nodes work in initial deploy phase, meanwhile mobile sensor nodes switch to sleeping mode. We make some assumptions as follows:

- Both static and mobile sensors are homogenous.

- Both static and mobile sensors have the same initial energy.

- Both static and mobile sensors have the same sensing radius. 
- There is no big obstacle in the network.

- The coordinates of the nodes are known.

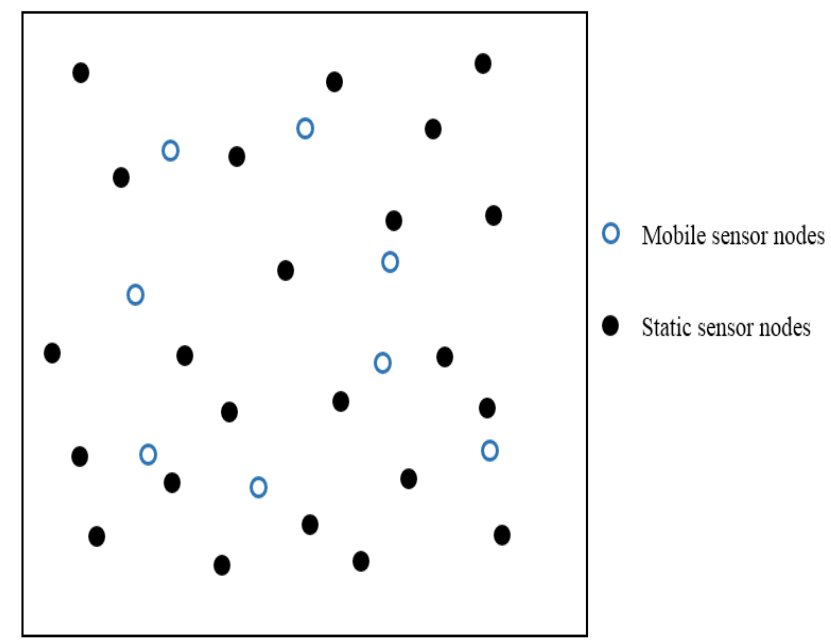

Figure 1 Network Model

\subsection{Coverage Hole Detection}

In traditional WSNs, sensor nodes are randomly deployed in the target area, and this deployment results in the unavoidable coverage hole problem. With the aim to fully cover the target area, coverage hole detection is required before deploy extra sensor nodes. In this paper, we divided the whole network into several grids and each grid has the same size. Then we calculate the coverage rate of each grid in order to identify the grid of low node density, as is shown in Figure 2.

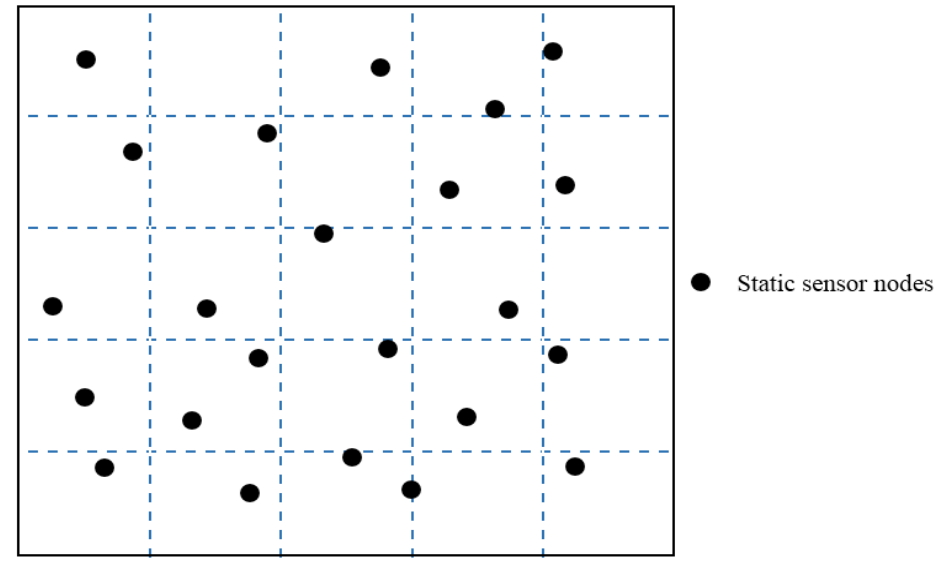

Figure 2 Grid Structure of WSNs

\subsection{Coverage Rate Calculation}

We deploy $m$ static sensors with sensing radius $r$. All sensors can be viewed as node set $G=\left\{g_{1}, g_{2}, g_{3}, \ldots, g_{n}\right\}$, where $g_{i}$ denotes node $i$ and its coordinate is $\left(x_{i}, y_{i}\right)$. For pixel $P\left(x_{p}, y_{p}\right)$ in grid, we can assume it is covered by node $g_{i}$ if it is within the sensing range of node $g_{i}$. The sensing rate $p\left(g_{i}, P\right)$ for pixel $P$ covered by $g_{i}$ is shown in formula (1). 


$$
p\left(g_{i}, P\right) \begin{cases}1, & d\left(g_{i}, P\right) \leq r \\ 0, & d\left(g_{i}, P\right)>r\end{cases}
$$

Here $d\left(g_{i}, P\right)=\sqrt{\left(x_{i}-x_{p}\right)^{2}+\left(y_{i}-y_{p}\right)^{2}}$ is the distance between pixel $P$ and node $g_{i}$. Meanwhile, pixel $P$ can be covered by several nodes. We can judge whether pixel $P$ is covered by nodes set $G$ in formula (2).

$$
p(G, P)=1-\prod_{g_{i} \in G}\left[1-p\left(g_{i}, P\right)\right](i=1,2,3, \ldots, n)
$$

The coverage rate in target area can be shown as:

$$
C O V=\frac{\sum_{P \in m \times n} p(G, P)}{m \times n}
$$

\subsection{Fitness Function}

For a mobile assist sensor network, moving distance is a necessary factor. We combine node moving distance with coverage rate as our fitness function $f(x)$, as is shown in formula (4). Here $\alpha$ and $\beta$ are adjustable parameters, and $\alpha+\beta=1$.

$$
f(x)=\frac{\alpha}{\sqrt{\left(x_{i n i}-x_{f i n}\right)^{2}+\left(y_{i n i}-y_{f i n}\right)^{2}}}+\beta \bullet(C O V)
$$

The initial position of mobile nodes is set as $\left(x_{i n i}, y_{i n i}\right)$ and its target position is set as $\left(x_{f i n}, y_{f i n}\right)$. We utilize particle swarm optimization algorithm to calculate the moving position for mobile sensors. In each step, we select particles' current position as mobile nodes' target position and then calculate their fitness function $f(x)$. This fitness function will further be used to compare with particles' individual and global optimal solutions later on.

\section{Our Proposed Algorithm}

\subsection{Initial Phase}

In this phase, $M$ static sensor nodes and $N$ mobile sensor nodes are randomly deployed in the sensor network. Static nodes switch into work mode in this step. Meanwhile, mobile nodes turn to sleep mode, they start to work after coverage holes are found.

\subsection{Grid Partition}

In this phase, we divide the whole network into same sized square grids to find sparse node regions. As is shown in Figure 2, the network is partitioned into 25 grids. Then, we calculate the coverage rate of each grid. If the coverage rate is less than $90 \%$, then we consider that the coverage rate can be improved in this grid $C_{i}(i \leq 25)$ and add it inside candidate grid set $C$; otherwise, we will skip this grid. At the end of this phase, a candidate grid set $C$ is selected, and we can see that there are coverage holes in this set. Then we deploy mobile sensors in the following phase among these grids. Grid partition process is shown in Figure 3. 


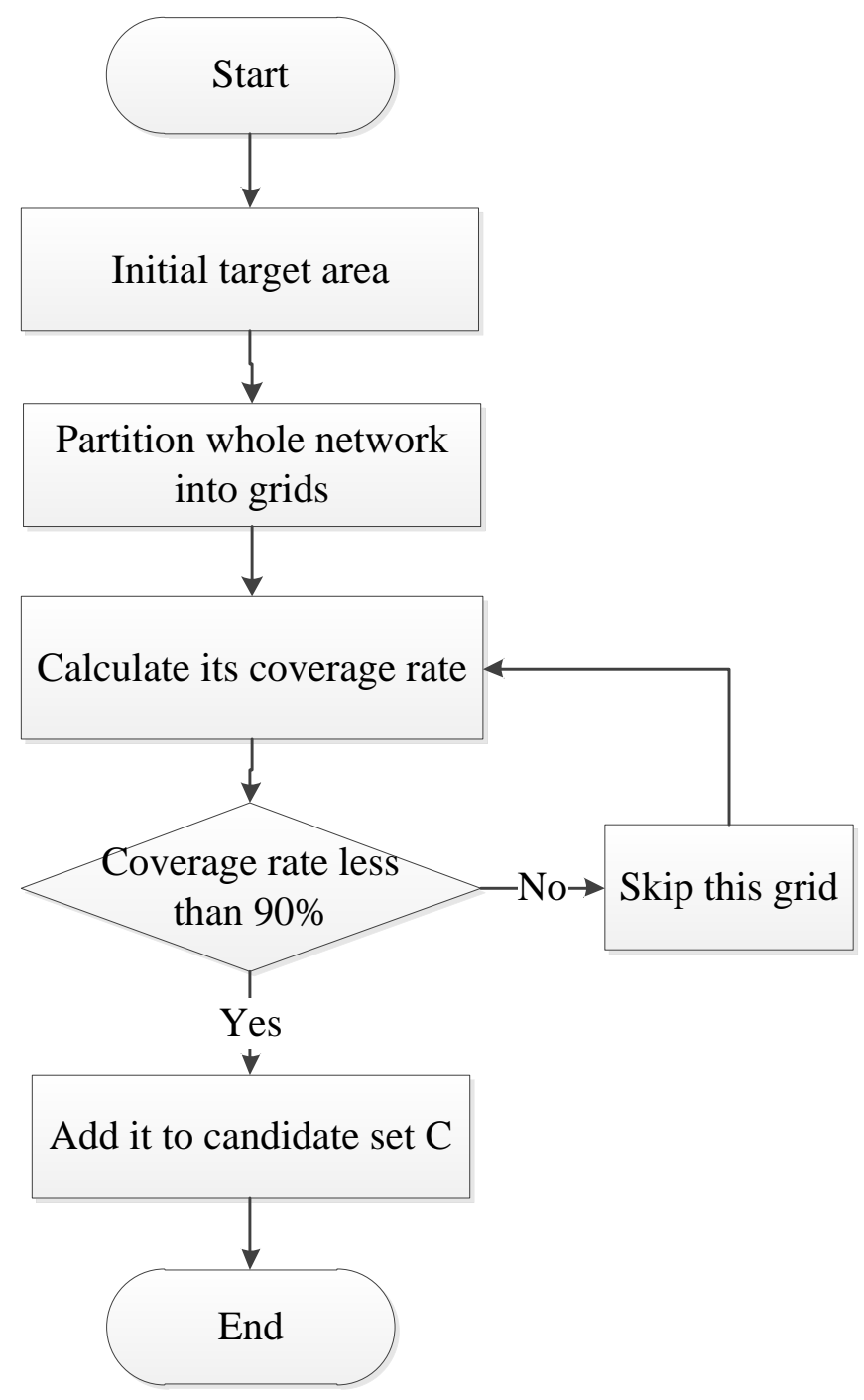

Figure 3 Flow chart of grid partition process

\subsection{Mobile Sensor Deployment}

After the candidate set $C$ is selected, we plan the moving position of mobile sensor nodes in order to cover these grids. We utilize the particle swarm optimization algorithm to calculate the optimal position. Then mobile sensors are awake and they move to the designated location. This is shown in Figure 4. 


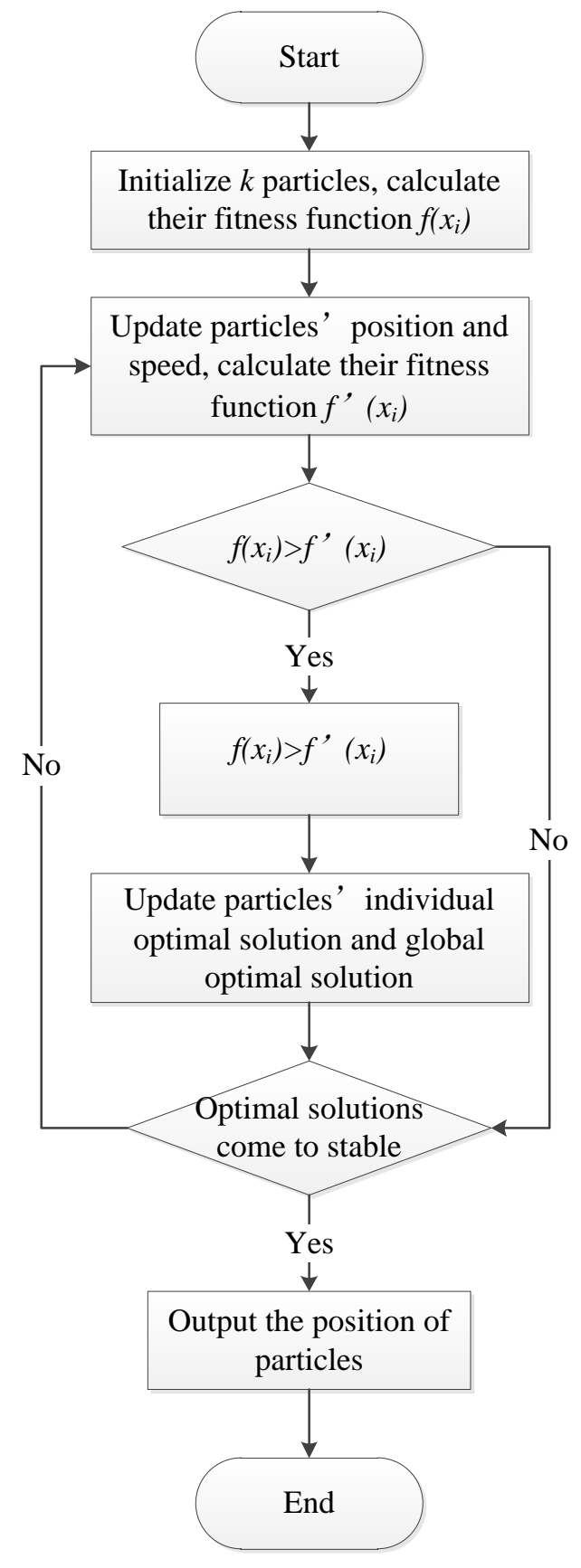

Figure 4 Flow chart of mobile sensor deployment

Mobile sensor deployment steps are shown below:

Step 1: We initialize $k$ particles $X\left(x_{1}, x_{2}, x_{3}, \ldots, x_{n}\right)$, and the search interval of $x_{i}(i=1,2,3, \ldots, n)$ is among the coordinate of candidate set $C$. The speed of particle $x_{i}$ is $v_{i}$, the individual optimal solution for particle $x_{i}$ is $p_{i}=\left(p_{i 1}, p_{i 2}, \ldots, p_{i n}\right)$ and the global optimal solution is $p_{g}=\left(p_{g 1}, p_{g 2}, \ldots, p_{g n}\right)$. Fitness function in formula (4) is $f(x)$.

Step 2: Each particle calculates the fitness function $f(x)$ and records its individual optimal solution and global optimal solution respectively.

Step 3: Each particles update its position and speed.

Step 4: Update the inertia weight of the PSO algorithm.

Step 5: Each particle calculates the fitness function $f(x)$, updates its individual optimal solution and global 
optimal solution.

Step 6: If particle's individual optimal solution and global optimal solution become stable, then output the position of particles and go to the end; else, go to Step 3.

Finally, the global optimal solution of particle is achieved as the best solution, and mobile sensor nodes move to their optimal position.

\section{Performance Evaluation}

To evaluate the performance of our proposed Mobile Assisted Coverage Hole Patching Scheme (MACHPS), we compare it with other two algorithms named CMPA [14] and CHHA [16]. Simulation is conducted with Matlab. The number of static sensor nodes is fixed and they remain static after deployment. The simulation parameters are shown in Table 1.

Table 1 Simulation Parameters

\begin{tabular}{ll}
\hline \hline Simulation Parameters & Values \\
\hline Network Size & $100 * 100 \mathrm{~m}^{2}$ \\
Static node deployment & Random \\
Number of static node & 35 \\
Number of mobile node & {$[0,12]$} \\
Sensing range & {$[3,8] \mathrm{m}$} \\
\hline
\end{tabular}

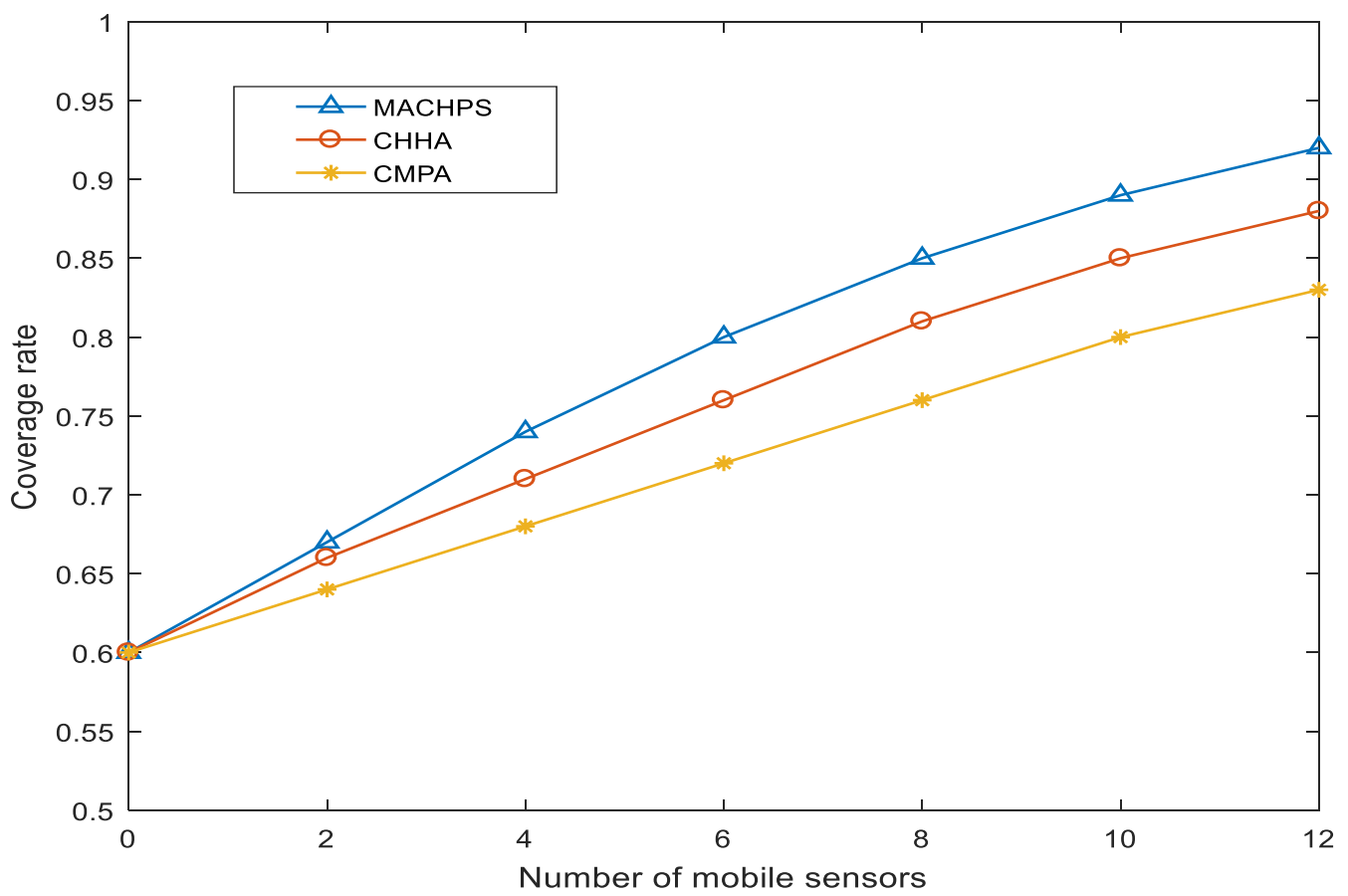

Figure 5 Comparison under different mobile sensor number

The purpose of our algorithm is to maximize the coverage rate and improve network performance. In Figure 5 , the sensing rang is 8 meters. We then add the number of mobile sensor nodes and compare coverage rate of our MACHPS algorithm with CHHA and CMPA algorithms. We can see that with the number of mobile sensor nodes increasing, the coverage rate grows with it. Coverage rate increases since more mobile sensor nodes heal coverage holes, and our MACHPS has better performance than the other two algorithms. This shows that our algorithm can effectively find coverage holes in sensor network. 
Sensing range is a significant factor in node coverage algorithms. We assume that the number of mobile sensor nodes is 12 and change sensing radius of nodes. We can see in Figure 6 that the coverage rate grows as nodes' sensing range increases. When the sensing range comes to a certain degree, coverage rate becomes stable. Compare with other two algorithms, our proposed algorithm performances better. This is because that sensor nodes are evenly deployed in our algorithm.

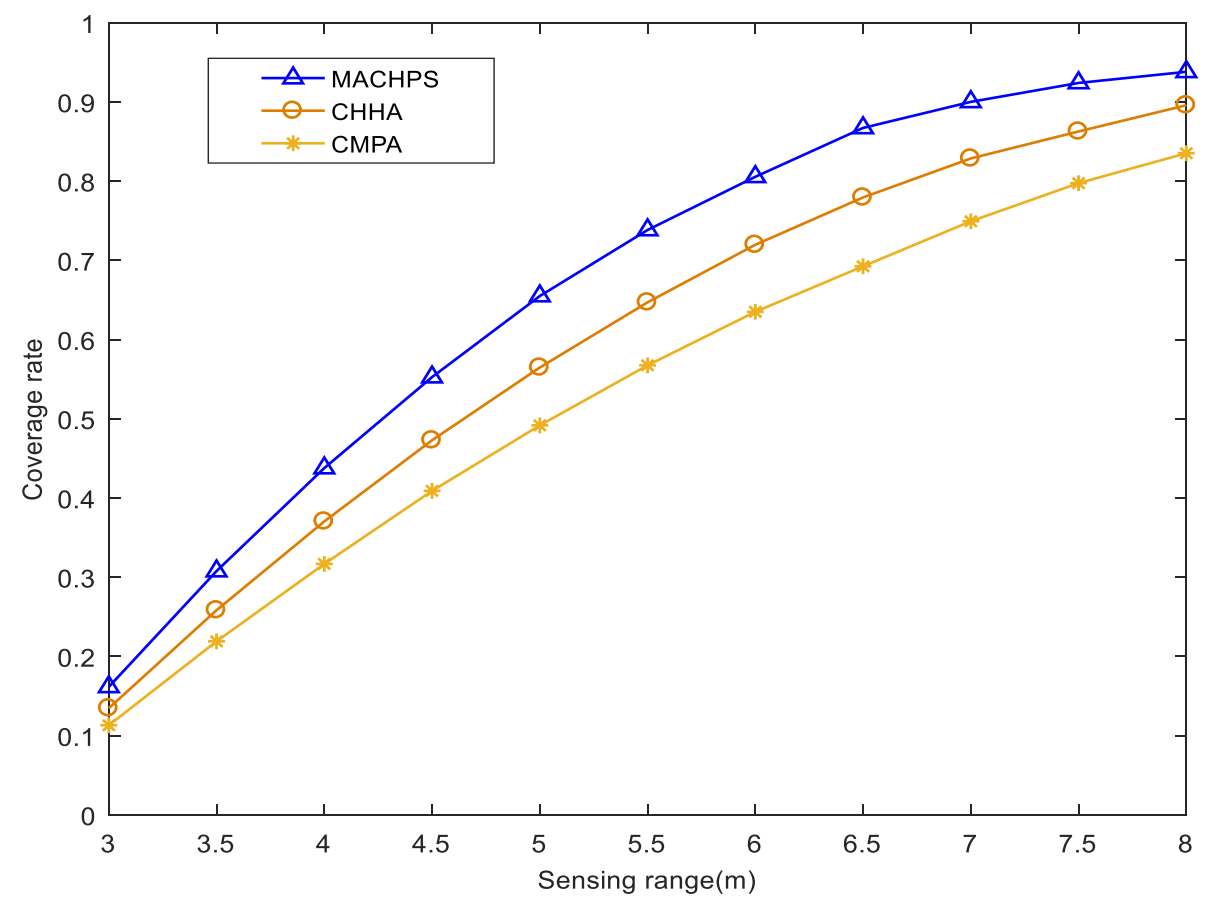

Figure 6 Comparison under different sensing range

Besides, we also calculate the average moving distance of mobile sensor nodes. We assume that nodes' sensing range is 8 meters. Then we can see from Figure 7 that when the number of mobile sensors is 2 , sensors move about 31 meters in our algorithm, meanwhile, other two algorithms' average moving distance is larger. As the number of mobile sensors increases, the average moving distance of our algorithm is much smaller as compared to the other two algorithms. Since mobile sensors are randomly deployed in initial phase, distance between nodes and coverage holes are shorter. 


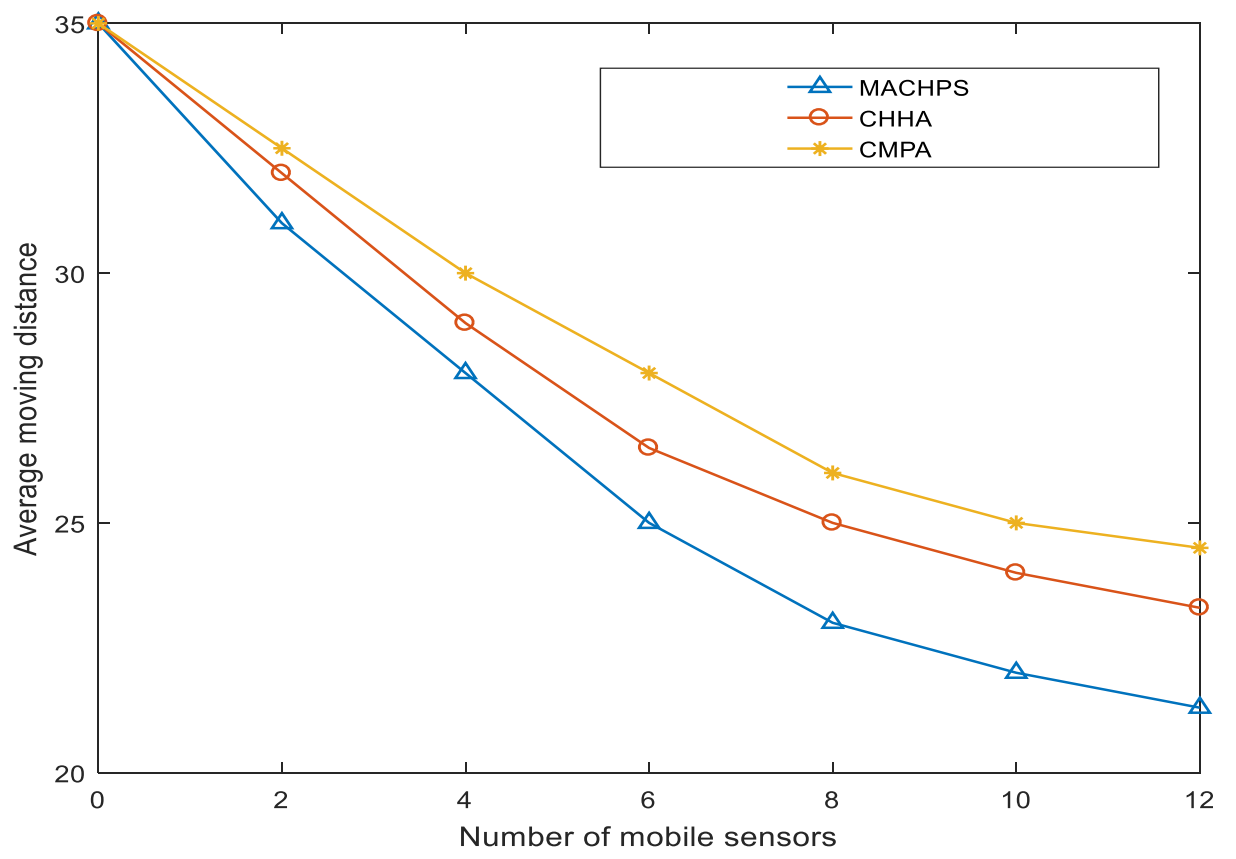

Figure 7 Comparison of average moving distance

\section{Discussion and open research issues}

Coverage control plays a vital role in WSNs, which can largely influence the performance of WSNs. Coverage problems can be further divided into three categories, namely point coverage, barrier coverage and area coverage problems. Though tremendous research effort has been concentrated on coverage problems in WSNs, there are still many research issues which need to be further explored.

1) Initial deployment strategies of sensor nodes: Generally, there are two strategies to deploy sensor nodes which are random deployment and planned deployment. Random deployment strategy is usually adopted in large scale WSNs, since sensor nodes can be quickly in target area by using aircraft. However, this kind of deployment cannot uniformly spread sensor nodes, which can give rise to coverage hole problem. Some research focuses on delete redundant nodes and adds extra sensor nodes on vital areas to alleviate this problem. This aspect can be further studied for large scale WSNs.

2) Sensor nodes mobility: For mobile WSNs, all sensor nodes can move after initial deployment. In such circumstance, node scheduling algorithms are necessary to cover target areas. This approach mainly considers the sensors' movement distance and energy efficiency. Also, mobile senor nodes can be adopted in static WSNs, for mobile sensor nodes, they can move to coverage hole after static sensor nodes are deployed in target area.

3) Energy efficiency: Energy efficiency is an essential issue in building a WSN. Long distance transmission, frequent routing reconfiguration and communication overload can give rise to extra energy cost. To alleviate this problem, sensor nodes deployment and scheduling algorithms are of vital importance.

4) Moving distance of mobile sensors: For those coverage algorithms with mobile sensors, the moving distance for sensors should be carefully considered and designed when scheduling mobile sensors.

\section{Conclusions}

In this paper, we proposed a mobile assisted node deployed scheme based on particle swarm optimization for WSNs. We first randomly deploy sensor nodes, and static nodes start to work in this step. For mobile sensor nodes, they switch to sleep model in initial phase. Then we divide the whole network into same sized square grids to find sparse node regions. Grids with lower coverage rate are selected as candidate grids, and they will be covered by mobile sensors later. Finally, we utilize PSO algorithm to find the optimal mobile sensor deployment position. Simulation results show that our proposed algorithm is superior compared with other two popular algorithms. 


\section{Acknowledgements}

This research work is supported by the NSFC (61772454), and by the open research fund of Key Lab of Broadband Wireless Communication and Sensor Network Technology (Nanjing University of Posts and Telecommunications), Ministry of Education. It is also supported by Industrial Core Technology Development Program (10049079, Development of Mining core technology exploiting personal big data) funded by the Ministry of Trade, Industry and Energy (MOTIE), Korea. Prof. Hye-jin Kim is the corresponding author.

\section{References}

[1] Akkaya K, Younis M. A survey on routing protocols for wireless sensor networks [J]. Ad hoc networks, 3(3), 2005, 325-349.

[2] Yick J, Mukherjee B, Ghosal D. Wireless sensor network survey [J]. Computer networks, 52(12), 2008, 2292-2330.

[3] Song W Z, Huang R, Xu M, et al. Design and deployment of sensor network for real-time high-fidelity volcano monitoring [J]. IEEE Transactions on Parallel and Distributed Systems, 21(11), 2010, 1658-1674.

[4] Younis M, Akkaya K. Strategies and techniques for node placement in wireless sensor networks: A survey [J]. Ad Hoc Networks, 6(4), 2008, 621-655.

[5] Wang X, Han S, Wu Y, et al. Coverage and energy consumption control in mobile heterogeneous wireless sensor networks [J]. IEEE Transactions on Automatic Control, 58(4), 2013, 975-988.

[6] Senouci M R, Mellouk A, Asnoune K, et al. Movement-assisted sensor deployment algorithms: A survey and taxonomy [J]. IEEE Communications Surveys \& Tutorials, 17(4), 2015, 2493-2510.

[7] Mahboubi H, Moezzi K, Aghdam A G, et al. Distributed deployment algorithms for efficient coverage in a network of mobile sensors with nonidentical sensing capabilities [J]. IEEE Transactions on Vehicular Technology, 63(8), 2014, 3998-4016.

[8] Senouci M R, Mellouk A, Asnoune K, et al. Movement-assisted sensor deployment algorithms: A survey and taxonomy[J]. IEEE Communications Surveys \& Tutorials, 17(4), 2015, 2493-2510.

[9] Mini S, Udgata S K, Sabat S L. Sensor deployment and scheduling for target coverage problem in wireless sensor networks [J]. IEEE Sensors Journal, 14(3), 2014, 636-644.

[10] Senouci M R, Mellouk A, Assnoune K. Localized movement-assisted sensor deployment algorithm for hole detection and healing [J]. IEEE Transactions on parallel and distributed systems, 25(5), 2014, 1267-1277.

[11] Liao Z, Wang J, Zhang S, et al. Minimizing movement for target coverage and network connectivity in mobile sensor networks [J]. IEEE Transactions on Parallel and Distributed Systems, 26(7), 2015, 1971-1983.

[12] Chen J, Shen E, Sun Y. The deployment algorithms in wireless sensor networks: A survey [J]. Information Technology Journal, 8(3), 2009, 293-301.

[13] Rakavi A, Manikandan M S K, Hariharan K. Grid based mobile sensor node deployment for improving area coverage in Wireless Sensor Networks[C]//Signal Processing, Communication and Networking (ICSCN), 2015 3rd International Conference on. IEEE, 2015, 1-5.

[14] Li F, Xiong S, Wang L. Recovering coverage holes by using mobile sensors in wireless sensor networks[C]//Computational Intelligence and Security (CIS), 2011 Seventh International Conference on. IEEE, 2011, 746-749.

[15] Abolhasan M, Maali Y, Rafiei A, et al. Distributed Hybrid Coverage Hole Recovery in Wireless Sensor Networks [J]. IEEE Sensors Journal, 16(23), 2016, 8640-8648.

[16] Nguyen D T, Nguyen N P, Thai M T, et al. An optimal algorithm for coverage hole healing in hybrid sensor networks[C]//Wireless Communications and Mobile Computing Conference (IWCMC), 2011 7th International. IEEE, 2011, 494-499.

[17] Zhang Y, Sun X, Wang B. Efficient algorithm for k-barrier coverage based on integer linear programming [J]. China Communications, 13(7), 2016, 16-23.

[18] Wang B, Gu X, Ma L, et al. Temperature error correction based on BP neural network in meteorological wireless sensor network [J]. International Journal of Sensor Networks, 23(4), 2017, 265-278.

[19] Zhang J, Tang J, Wang T, et al. Energy-efficient data-gathering rendezvous algorithms with mobile sinks for wireless sensor networks [J]. International Journal of Sensor Networks, 23(4), 2017, 248-257.

[20] Yasmine-Derdour, Bouabdellah-Kechar, Fayçal- Khelfi M. Using mobile data collectors to enhance energy efficiency and reliability in delay tolerant wireless sensor networks [J]. Journal of Information Processing Systems, 12(2), 2016, 275-294. 
[21] Gupta G P, Misra M, Garg K. An energy efficient distributed approach-based agent migration scheme for data aggregation in wireless sensor networks [J]. Journal of Information Processing Systems, 11(1), 2015, $148-164$.

[22] Jaiswal P, Sinha A. Stable geographic forwarding with link lifetime prediction in mobile adhoc networks for battlefield environment [J]. Human-centric Computing and Information Sciences, 6(1), 2016, 22.

[23] Zhu H, Xiao F, Sun L, et al. R-TTWD: Robust Device-free Through-The-Wall Detection of Moving Human with WiFi [J]. IEEE Journal on Selected Areas in Communications, 35(5), 2017, 1090-1103.

[24] Xiao F, Sha L T, Yuan Z P, et al. VulHunter: A Discovery for unknown Bugs based on Analysis for known patches in Industry Internet of Things [J]. IEEE Transactions on Emerging Topics in Computing, DOI:10.1109/TETC.2017.2754103, Published online, 2017, 1-13. 